Supporting Information

\title{
Tailoring morphology compatibility and device stability by adding PBDTTPD-COOH as third component to fullerene- based polymer solar cells
}

Dan Yang,${ }^{\dagger}$ Bing Cao,$\stackrel{\star}{ }$ Volker Körstgens,${ }^{\dagger}$ Nitin Saxena, ${ }^{\dagger}$ Nian Li,${ }^{\dagger}$ Christoph Bilko,

${ }^{\dagger}$ Sebastian Grott, ${ }^{\dagger}$ Wei Chen,${ }^{\dagger}$ Xinyu Jiang,,${ }^{\dagger}$ Julian Eliah Heger, ${ }^{\dagger}$ Sigrid Bernstorff, $\uparrow$ Peter Müller-Buschbaum ${ }^{\dagger, \&, *}$

${ }^{\dagger}$ Lehrstuhl für Funktionelle Materialien, Physik-Department, Technische Universität

München. James-Franck-Str. 1, 85748 Garching, Germany

Hniversity of Alberta, Department of Chemistry, Edmonton, AB T6G 2G2, Canada

`Elettra-Sincrotrone Trieste S.C.p.A., Strada Statale 14-km 163.5 in AREA Science

Park, Basovizza, 34149 Trieste, Italy

$\S$ Heinz Maier-Leibnitz Zentrum (MLZ), Technische Universität München, Lichtenbergstr. 1, 85748 Garching, Germany

*Corresponding author: muellerb@ph.tum.de 

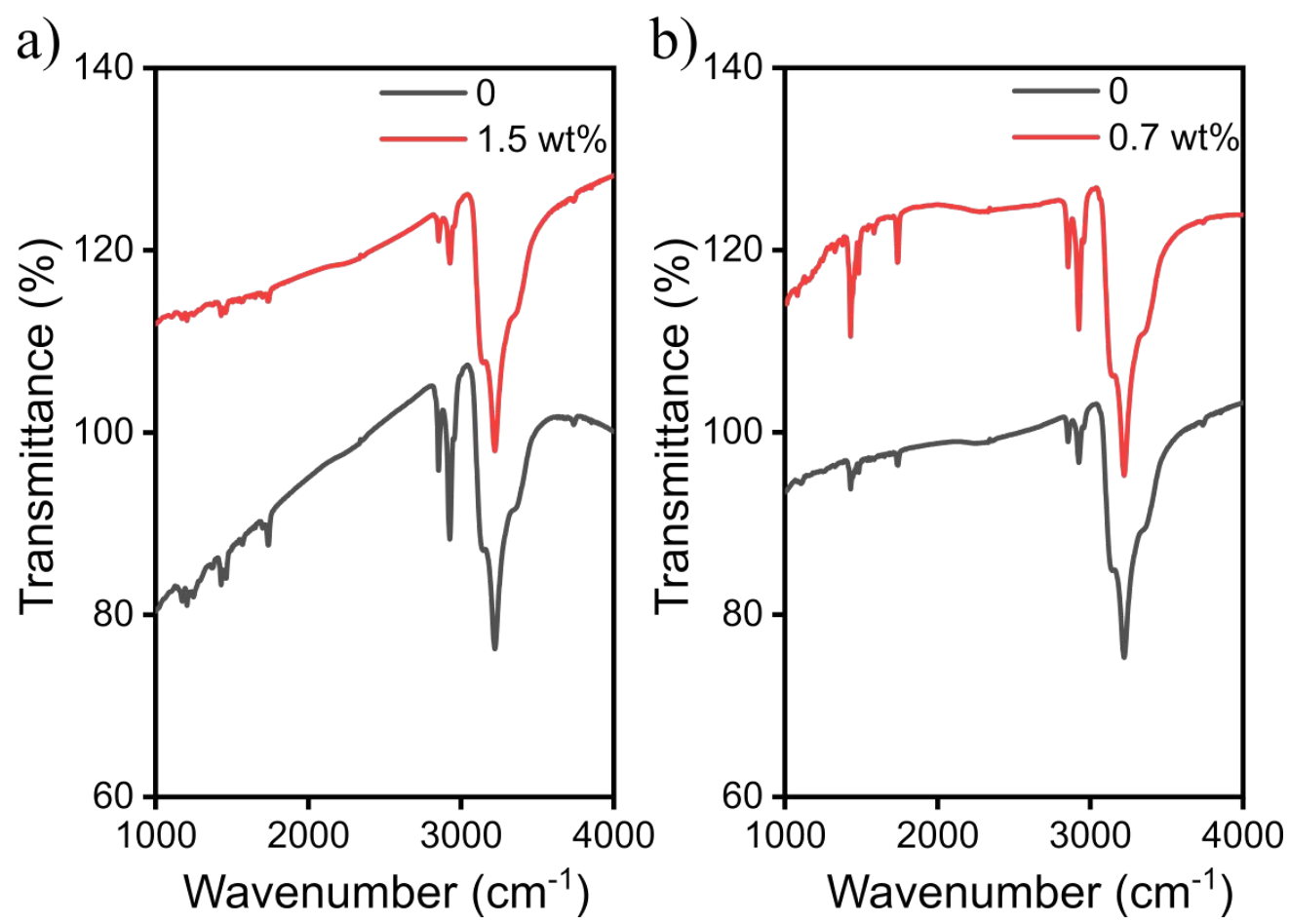

Figure 1. FTIR spectra of a) PTB7-Th:PC ${ }_{71} \mathrm{BM}$ binary film and PTB7-Th:PC ${ }_{71} \mathrm{BM}$ ternary film, b) PffBT4T-2OD:PC ${ }_{71} \mathrm{BM}$ binary film and PffBT4T-2OD:PC ${ }_{71} \mathrm{BM}$ ternary film. 

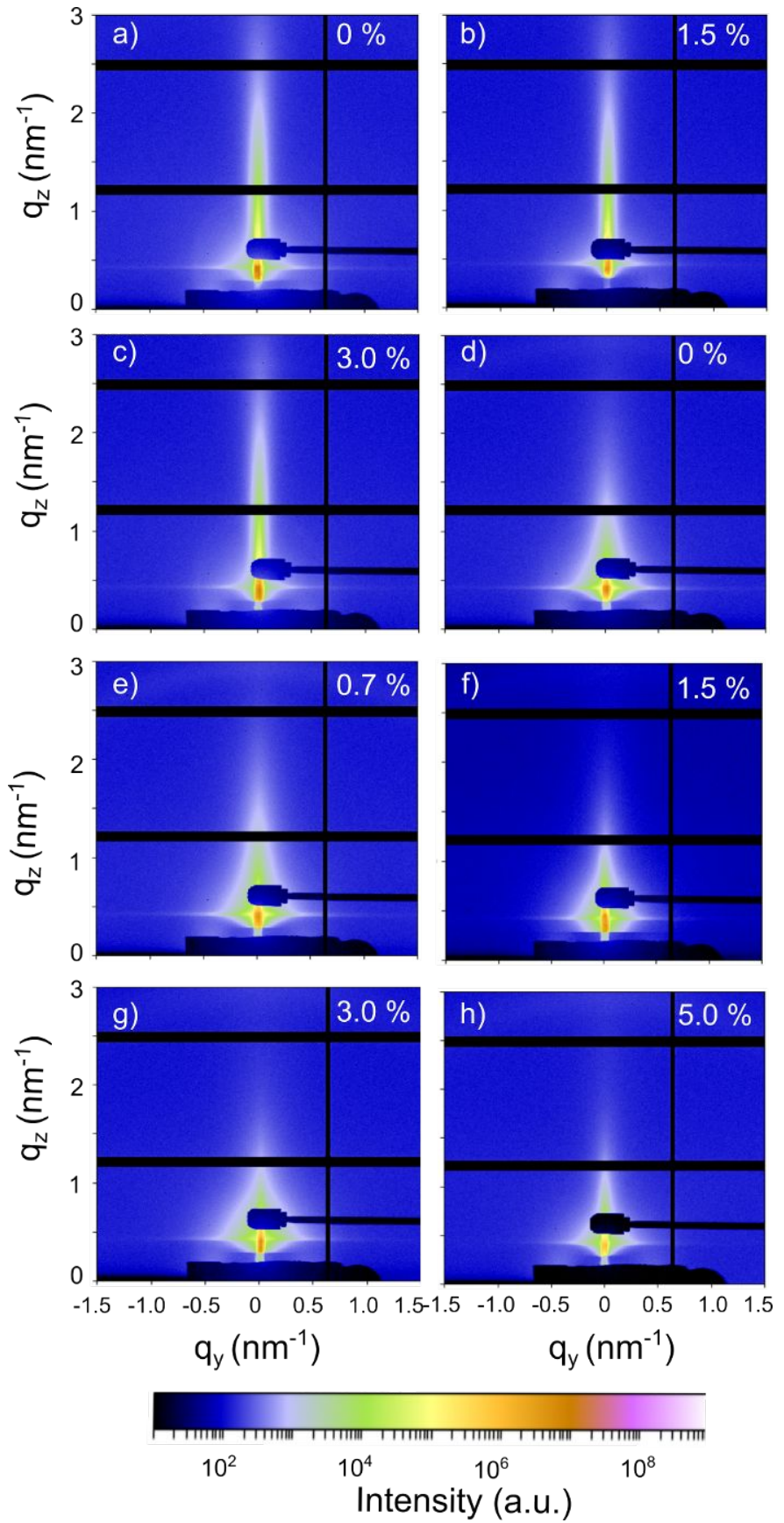

Figure S2. 2D GISAXS data of BHJ films: a)-c) PTB7-Th:PC ${ }_{71} B M$ and d)-h) PffBT4T-2OD:PC ${ }_{71} \mathrm{BM}$ films with different blend ratio of PBDTTPD-COOH. The specular peak is shielded with a rectangular beamstop. The black vertical and horizontal stripes are intermodal gaps of the detector. 

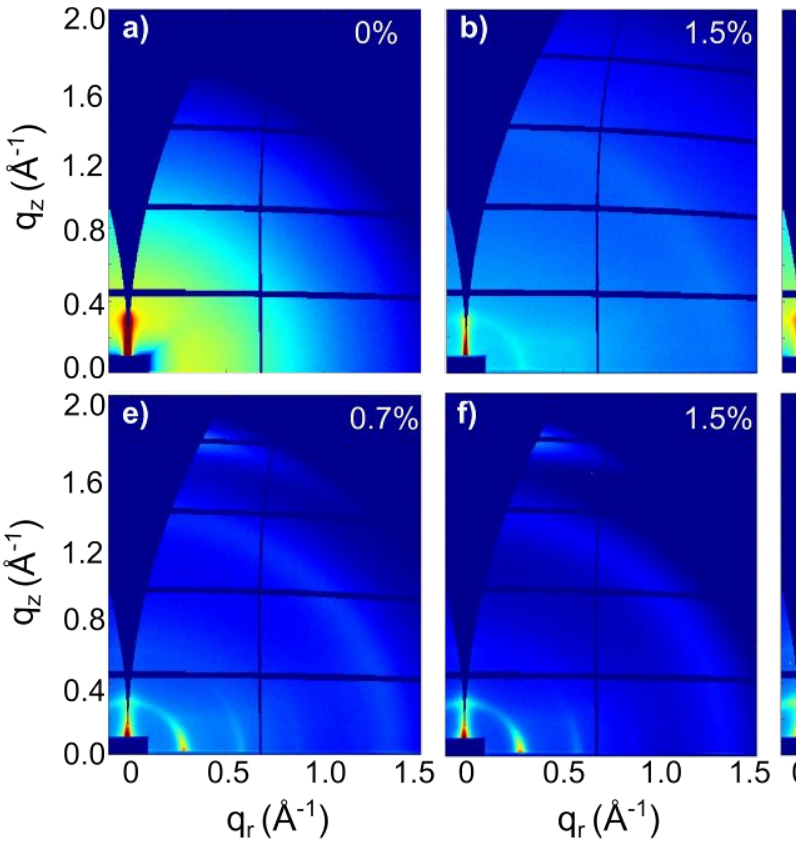

$\mathrm{q}_{\mathrm{r}}\left(\AA^{-1}\right)$

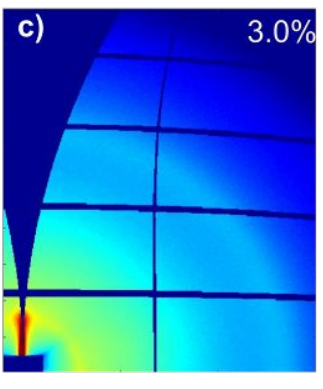

g)

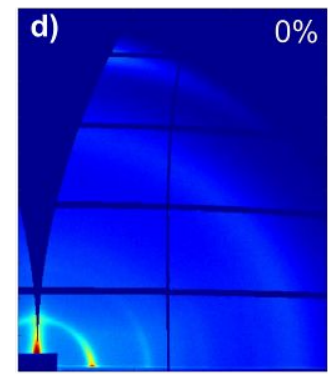

h)

$5.0 \%$

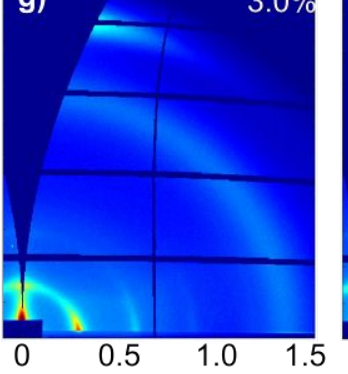

$\mathrm{q}_{\mathrm{r}}\left(\AA^{-1}\right)$

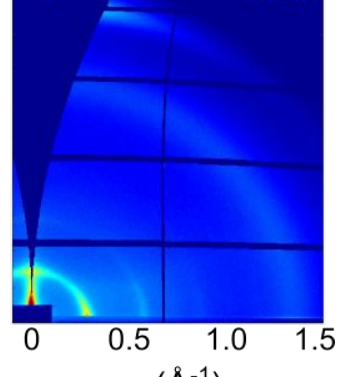

$\mathrm{q}_{\mathrm{r}}\left(\AA^{-1}\right)$

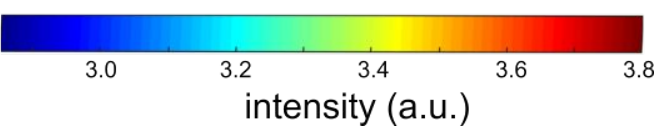

Figure S3. 2D GIWAXS data of BHJ films: a)-c) PTB7-Th:PC ${ }_{71} B M$ and d)-h) PffBT4T-2OD:PC ${ }_{71}$ BM films with different ratios of PBDTTPD-COOH. The vertical and horizontal black stripes are due to the inter-module gaps of the detector. 

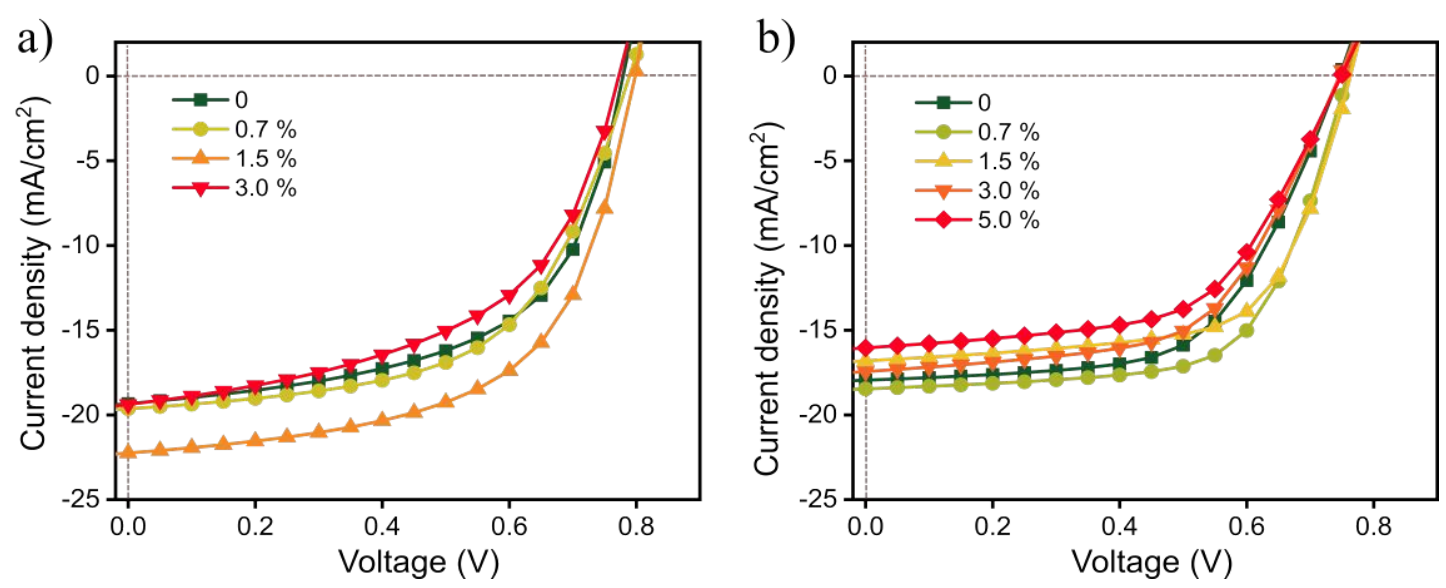

Figure S4. Representative $J-V$ curves of a) PTB7-Th:PC 71 BM devices and b) PffBT4T2OD: $\mathrm{PC}_{71} \mathrm{BM}$ devices with different ratios of PBDTTPD-COOH as indicated. 

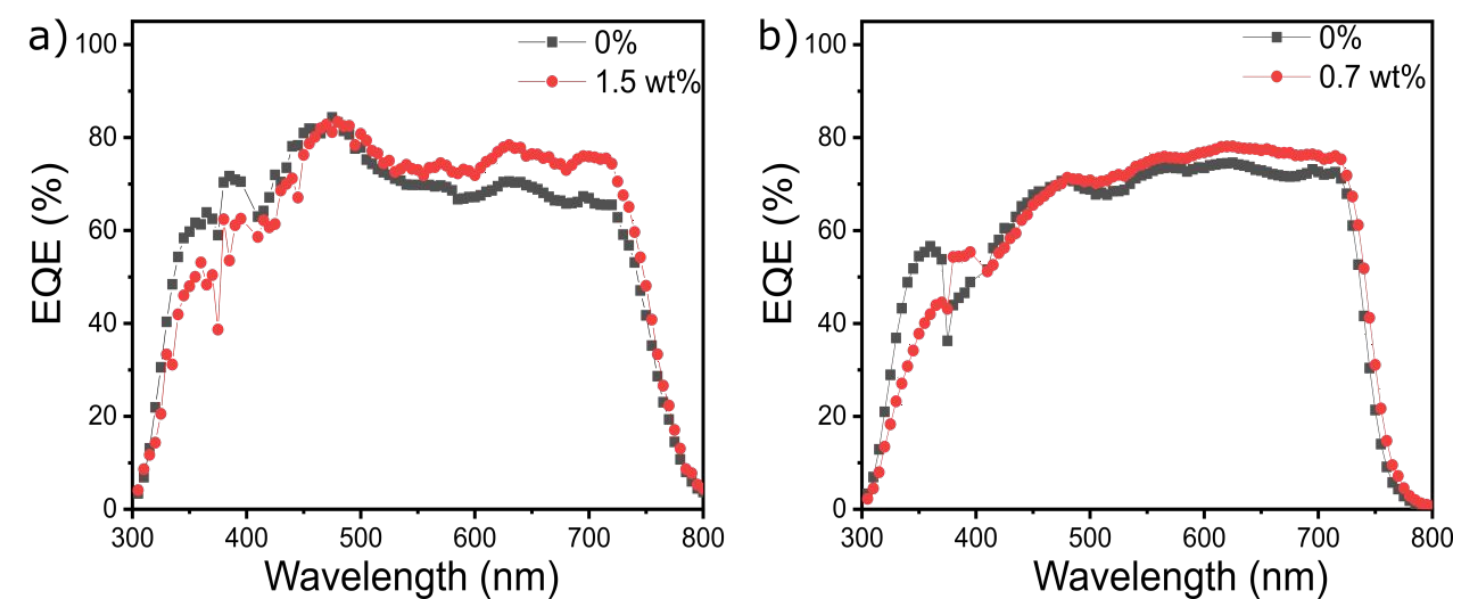

Figure S5. EQE spectra of a) $\mathrm{PTB} 7-\mathrm{Th}: \mathrm{PC}_{71} \mathrm{BM}$ reference cell (black) and optimal ternary device (red) and of b) PffBT4T-2OD:PC ${ }_{71} \mathrm{BM}$ reference cell (black) and optimal ternary device (red). 